Health \& Medicine | Aubrey Husak \& Matthew Bair

\title{
Bidirectional relationship between chronic pain and insomnia
}

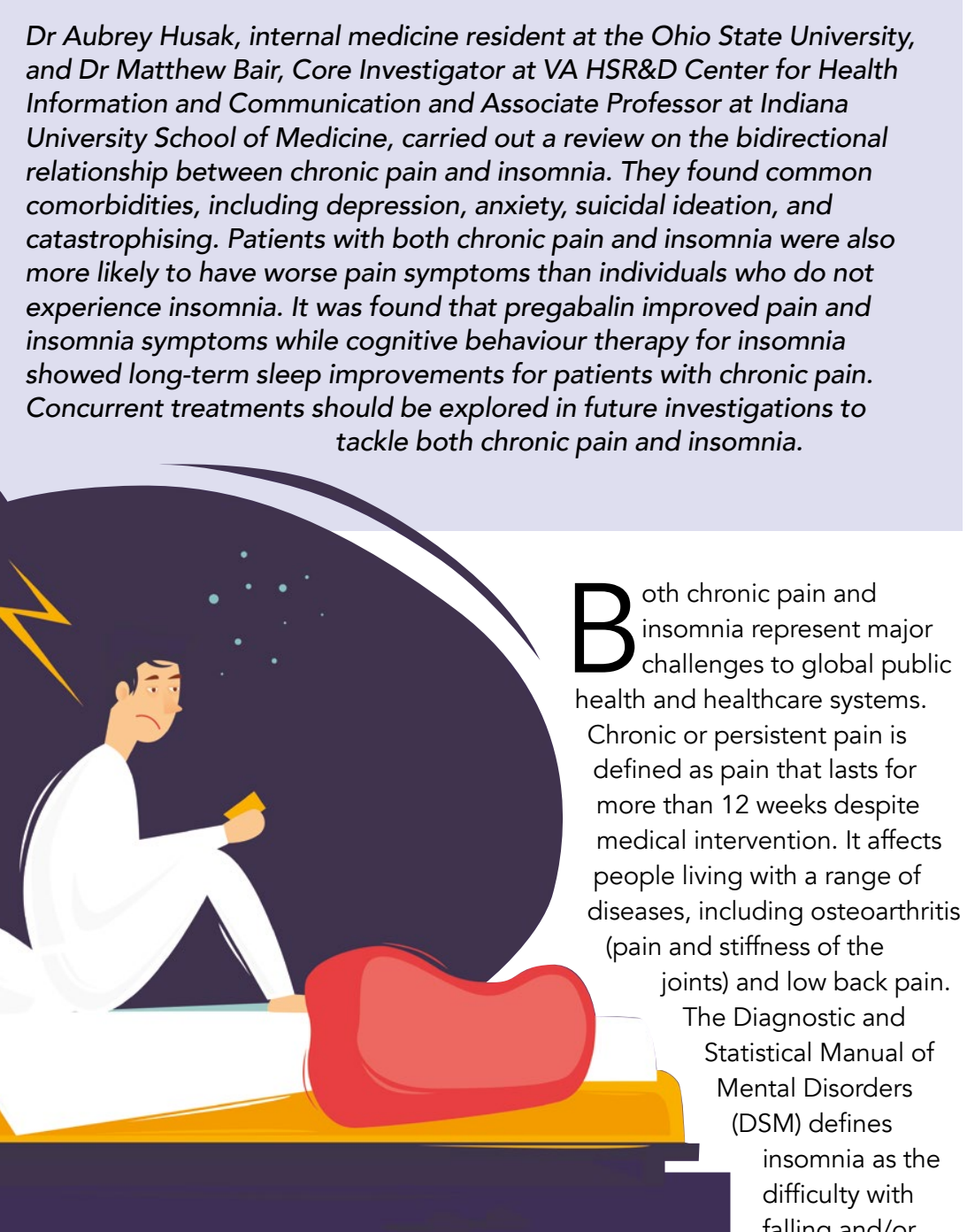

PODIIS/Sutterstock.com staying asleep, which results in a poor quality of sleep. Frequent transient wakefulness and prolonged periods of wakefulness can deeply impair an individual's day-to-day schedule. experience sleep disturbances for at least three days per week for at leas three months. The prevalence of chronic pain has been estimated to exist in 17 $25 \%$ of the general population, whereas approximately $10-15 \%$ of individuals live

24-32\% of patients with chronic pain aso report experiencing insomnia, as proportion of individuals in the general population. In addition, when insomnia is more broadly defined, the incidence of individuals living with both chronic pain and insomnia increases to a range of $40-80 \%$. This signifies that patients with chronic pain are more likely to exhibit symptoms of insomnia.

eople experiencing chronic pain and insomnia are more likely to have higher incidences of depression, anxiety, and fatigue. The strong relationship between chronic pain and insomnia, along with similar comorbidities, require further investigation to elucidate better treatment for this patient population.
Previously, it was assumed that insomnia is directly caused by chronic pain and alleviation of chronic pain will alss However, researchers recently theorised a bidirectional relationship, which states that chronic pain and insomnia exist together and can exacerbate each other.

\section{LITERATURE REVIEW}

Dr Aubrey Husak, internal medicine esident at the Ohio State University, and Dr Matthew Bair, Core Investigator at VA HSR\&D Center for Health Information and Communication and Associate Professor at Indiana University School of Medicine, set out to write a review that answers three questions to fill in the gaps on the relationship 1) How chronic pain and insomnia: How does concun and insonic pain and pain duration in patients with chronic pain?

2) What are the con and pain-related symptoms in options for both chronic pain and insomnia?

Two databases of research papers, OVID Medline and PubMed, were searched from 1946 to June 2017 for the topics 'chronic pain' and 'sleep disorders', with an emphasis on treatment. This search yielded 313 articles, but only 33 were included after eligibility checks. Partipia for insomna were included in the review, as these individuals can still experience symptoms that are lifedebilitating However, other related medical conditions such as sleep apnea and restless leg syndrome were not included in the review. The term chronic pain was broadly searched for, which yielded conditions like fibromyalgia (a long-term condition that causes widespread pain all over the body; thought to be caused by the way the nervous system processes pain signals) and osteoarthritis. Only randomised controlled trials that assess the effectiveness of treatments were included.

Dr Husak and Dr Bair's review found that there was a bidirectional relationship
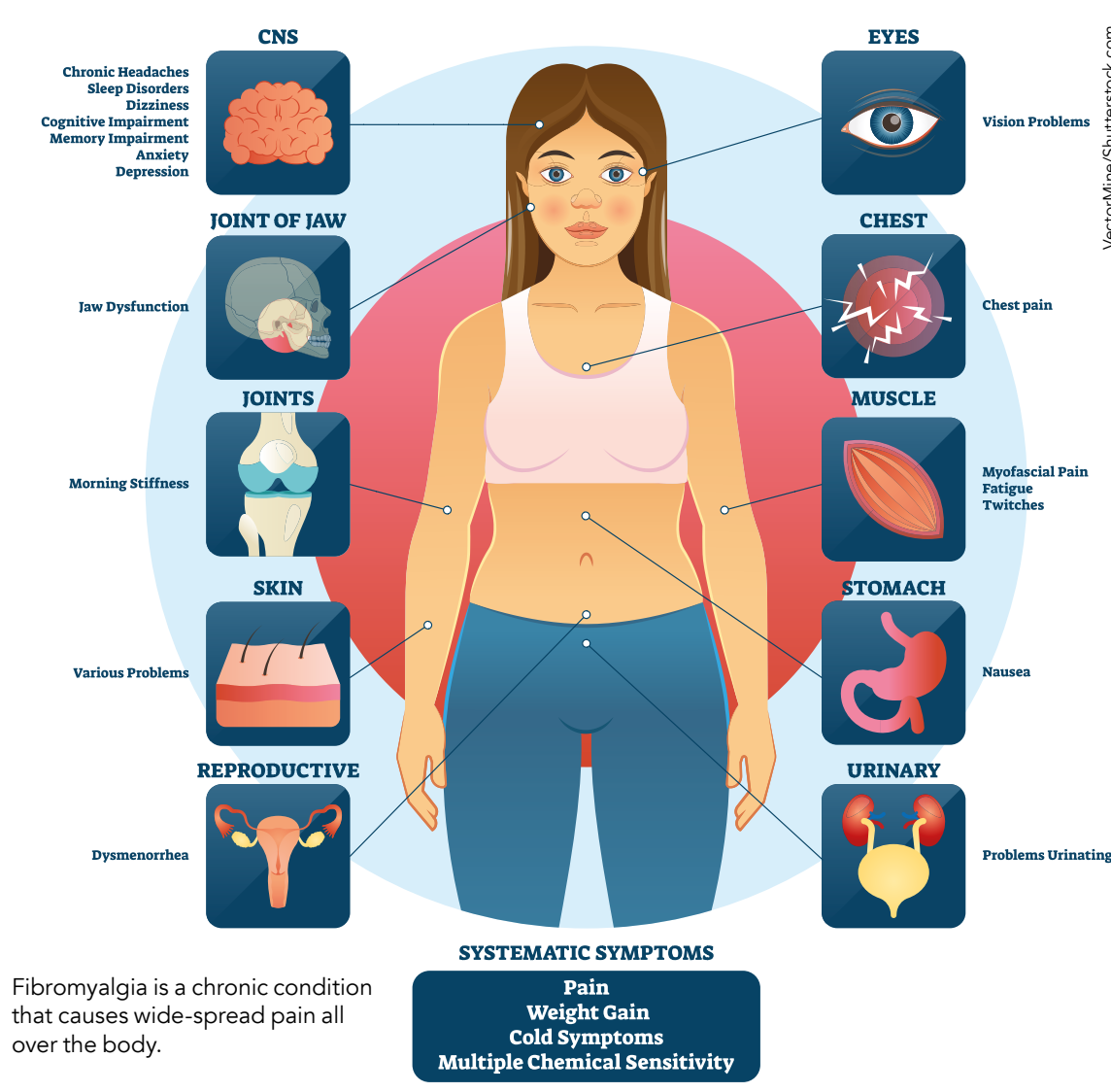

Fibromyalgia is a chronic condition
that causes wide-spread pain all

Resolution of sleep disturbances was more likely to be followed by reports of lower pain intensities.

between insomnia and chronic pain. Better sleep quality was correlated wit and predicted better improvement in chronic pain. Resolution of sleep disturbances was more likely to be followed by reponts of lower pain with disability or limitas ans correlated activity Individuals living with both chronic pain and insomnia were more likely to suffer from mental health issues, such as depression, anxiety

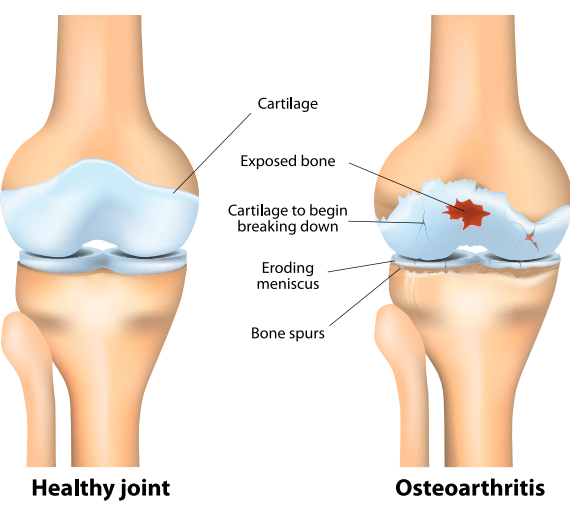

egative mood, suicidal thoughts, and catastrophising (viewing a situation as worse than it actually is). Previously, a chature review found hat patients with chronic pain also suffer from depressive symptoms, and hat chronic pain ( pain, and insomnia share similar biological pathways, it is not surprising diseases are comorbid with

\section{TREATMENT OPTIONS} The most studied pharmacological with fibromyalgi was pregabalin (a medication that and pain disorders; it exerts an inhibitory modulation of neurona excitability, reducing the release of neurotransmitters associated with pain Individuals who received pregabalin pain and insomnien pain and insomnia symptoms. Other in both conditions include mrement 


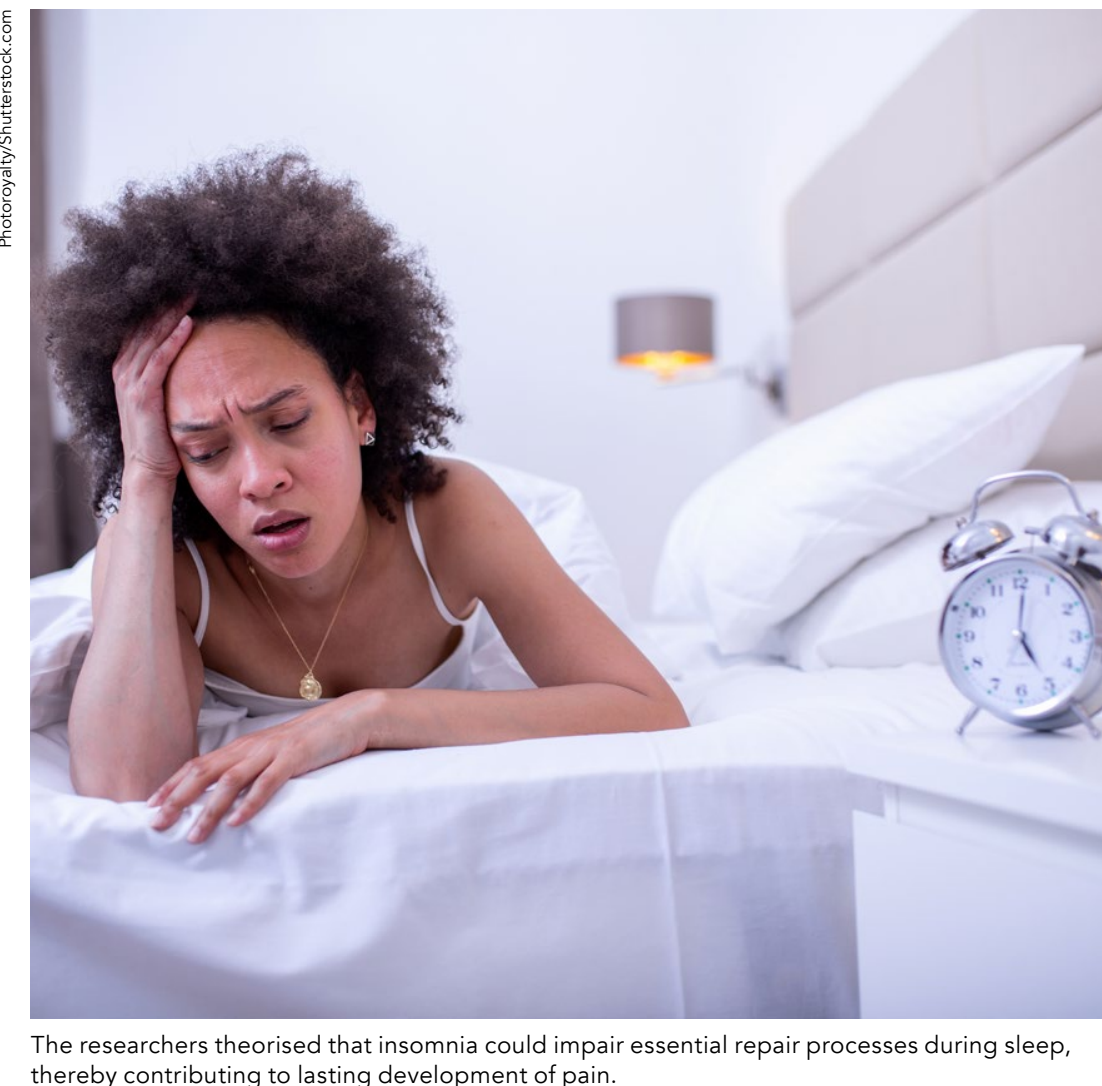

A combination of pharmacological and non-pharmacological treatments is a promising avenue to alleviate the symptoms and co-occurrence of both conditions.

eszopiclone (a sedative-hypnotic used to treat insomnia by binding to ABA receptar) and broxen (a

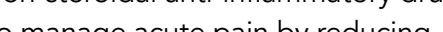
prostaglandin synthesis).

Cognitive behaviour therapy (CBT) was the most common non-pharmacologica treatment that was studied. This is a type of therapy that encourages an alternative way of thinking and behaving to manage an individual's problem, rooted in the fact that feelings and actions are interconnected. CBT for insomnia was found to improve sleep by decreasing the amount of time taken to fall asleep and increasing the duration of sleep. Reported pain was lso mildy improved. For CBT that aims to treat both insomnia and chronic pain investigations that reported positive

treatment for insomnia but no significant improvement for managing chronic pain. Along with CBT, pharmacologica also be administered together and investigated in future studies.

The review conducted by Dr Husak and Dr Bair supports the hypothesis of a bidirectional relationship between chronic pain and insomnia: individuals living with both conditions have a more severe pain experience and longer lasting symptoms, and additional comorbidities, such as mental health problems. The researchers theorised that insomnia could impair essential repair processes during sleep, thereby contributing to lasting development of pain related to dopamine and opioid signalling pathways. Tradition approaches aim po treat insomnia though the number of individuals We high. Moreover there is limited understanding of the biological relationship between both conditions. Therefore, further studies need to elucidate a concurrent treatment option for individuals with both conditions, so symptoms can be more effectively alleviated for this patient population.

A limitation of this study was that only papers in two databases, OVID Medline and PubMed, were searched for, which may result in some important research not included in this review. A metaanalysis, which combines and measures the statistical significance across various thdies, was also not conducted dur to the dire rences in definitions of and outcomes A challenge faced by researchers when conducting insomnia investigations is the lack of standardisation across different studies, as the categorisation and diagnosis of insomnia may vary due to differences in subjective and objective sleep quality measures. A variety of methods are utilised when assessing insomnia, such as polysomnography (a test that measures brain waves, blood oxyge levels, breathing, heart rate, and

movements of eye and leg), actigraphy (a device worn to monitor sleep cycles). and self-reports. Due to this subjectivity and heterogeneity in methodology, it is

\section{CONCLUSION}

In conclusion, this review emphasises the importance of assessing insomn and pain in individuals living with both pharmacologication of pharmacological treatments is a promising avenue to alleviate the symptoms and co-occurrence of both conditions. It also has the potential to decrease the severity, duration and comorbidities associated with these conditions, such as mental health problems. Suggested avenues for further investigations include cannabinoids and Internet-based CBT. Moreover, chronic pain is still inadequately treated, which calls for comorbid conditions and conging comorbid condifions and concurrenty

\section{Behind the Research Q \\ Dr Aubrey Husak ed/departments/internal-medicine/our-people/ residents

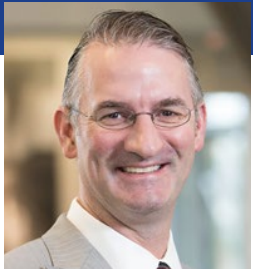 \\ Dr Matthew J. Bair \\ E: mbair@iupui.edu \\ T: $+13172879469 \quad$ T: +13179882058 W: https:///www.regenstrief.org/person/matthew-j-bair/ \\ Research Objectives}

Drs Husk a d Bar ivestigate common comorbidities and symptoms in patients with both chronic pain and sleep disturbances.

Detail

Matthew J Bair

A Center for Heath

Communication (11-H)

481 W. $10^{\text {th }}$ Street,

Indiana, USA 46202

Aubrey Husak, MD is an internal

\section{References}

Bair, M. J., Ang, D., Wu, J., Outcalt, S. D., et al. (2015). Evaluation of stepped care for chronic pain (ESCAPE) Veterans of the lraq and Afghanistan conticts: A randomized clinical trial. JAMA Internal Medicine, 175(5),
$682-689$.

Bair, M. J., Robinson, R. L., Katon, W. \& Kroenke, K. (2003). Depression and pain comorbidity. Archives of Internal Medicine, 163(20), 2344-2445.

Bair, M. J. \& Sanderson, T. R. (2011). Coanalgesics for chronic pain therapy: A narrative review. Postgraduate Medicine, 123(6), 140-150.

Husak, A. J. \& Bair, M. J. (2020). Chronic pain and sleep disturbances: A pragmatic review of their relationships, comorbidities, and treatments. Pain Medicine, 21(6), $1142-1152$

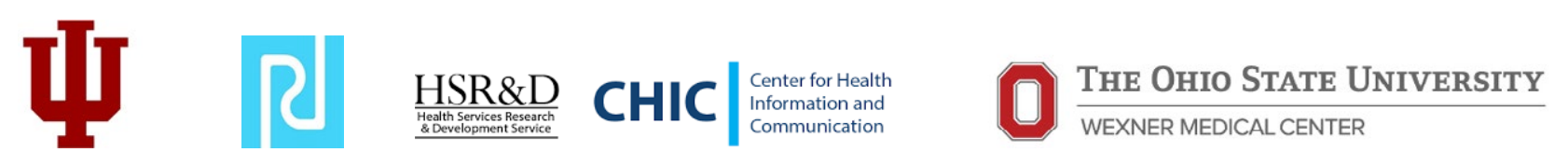

\section{Personal Response}

How can individual or group internet-based CBT benefit patients with both chronic pain and insomnia?

II Individual or group internet-based CBT can teach useful strategies and skills to patients to better cope
with their chronic pain and insomnia. A potential advantage of internet-based CBT is its greater reach to particularly important for patients in rural areas or those with transportation barriers. Patients may also like the convenience offered by Internet-based CBT in that they CBT is especially timely as patients may have increased pain, depression, anxiety, and sleep problems and need help with these conditions, but are fearful to seek
medical care in a clinical or hospital setting. patients that may not have access to clinicians trained to can access this treatment anytime or anywhere they wish. 